\title{
Detection of the tidal deformation of WASP-103b at $3 \sigma$ with CHEOPS (Corrigendum)
}

S. C. C. Barros ${ }^{1,2} \oplus$, B. Akinsanmi ${ }^{1,2,5}$, G. Bouée 3 , A. M. S. Smith ${ }^{4}$, J. Laskar ${ }^{3}$, S. Ulmer-Moll ${ }^{5}$, J. Lillo-Box 6 , D. Queloz ${ }^{5,7}$, A. Collier Cameron ${ }^{8}$, S. G. Sousa ${ }^{1}$, D. Ehrenreich ${ }^{5}$, M. J. Hooton ${ }^{9}$, G. Bruno ${ }^{10}$, B.-O. Demory ${ }^{9}$, A. C. M. Correia ${ }^{11}$, O. D. S. Demangeon ${ }^{1,2}$, T. G. Wilson ${ }^{8}$, A. Bonfanti ${ }^{12}$, S. Hoyer $^{13}$, Y. Alibert ${ }^{9}$, R. Alonso ${ }^{17,20}$, G. Anglada Escudé21,22, D. Barbato ${ }^{5}$, T. Bárczy ${ }^{23}$, D. Barrado ${ }^{6}$, W. Baumjohann ${ }^{12}$, M. Beck ${ }^{5}$, T. Beck ${ }^{9}$, W. Benz ${ }^{9}, 14$, M. Bergomi ${ }^{29}$, N. Billot ${ }^{5}$, X. Bonfils ${ }^{24}$, F. Bouchy ${ }^{5}$, A. Brandeker ${ }^{18}$, C. Broeg ${ }^{9,14}$, J. Cabrera ${ }^{4}$, V. Cessa ${ }^{9}$, S. Charnoz $^{25}$, C. C. V. Damme ${ }^{38}$, M. B. Davies ${ }^{26}$, M. Deleuil ${ }^{13}$, A. Deline ${ }^{5}$, L. Delrez ${ }^{27,28}$, A. Erikson ${ }^{4}$, A. Fortier ${ }^{9}, 14$, L. Fossati ${ }^{12}$, M. Fridlund ${ }^{31,19}$, D. Gandolfi ${ }^{32}$, A. García Muñoz ${ }^{39}$, M. Gillon ${ }^{27}$, M. Güdel ${ }^{33}$, K. G. Isaak ${ }^{34}$, K. Heng ${ }^{14,16}$, L. Kiss ${ }^{35,36}$, A. Lecavelier des Etangs ${ }^{37}$, M. Lendl ${ }^{5}$, C. Lovis $^{5}$, D. Magrin ${ }^{29}$, V. Nascimbeni' ${ }^{29}$, P. F. L. Maxted ${ }^{15}$, G. Olofsson ${ }^{18}$, R. Ottensamer ${ }^{33}$, I. Pagano ${ }^{10}$, E. Pallé ${ }^{17,20}$, H. Parviainen ${ }^{17,20}$, G. Peter ${ }^{4}$, G. Piotto ${ }^{29,30}$, D. Pollacco ${ }^{16}$, R. Ragazzoni ${ }^{29,30}$, N. Rando ${ }^{38}$, H. Rauer ${ }^{4,39,40}$, I. Ribas ${ }^{21,22}$, S. Salmon ${ }^{5}$, N. C. Santos ${ }^{1,2}$, G. Scandariato ${ }^{10}$, D. Ségransan ${ }^{5}$, A. E. Simon ${ }^{9}$, M. Steller ${ }^{12}$, Gy. M. Szabó ${ }^{41,42}$, N. Thomas ${ }^{9}$, S. Udry ${ }^{5}$, B. Ulmer ${ }^{43}$, V. Van Grootel ${ }^{27}$, and N. A. Walton ${ }^{44}$

\footnotetext{
${ }^{1}$ Instituto de Astrofísica e Ciências do Espaço, Universidade do Porto, CAUP, Rua das Estrelas, 4150-762 Porto, Portugal e-mail: susana.barros@astro.up.pt

${ }^{2}$ Departamento de Fisica e Astronomia, Faculdade de Ciencias, Universidade do Porto, Rua Campo Alegre, 4169-007 Porto, Portugal

${ }^{3}$ IMCCE, UMR8028 CNRS, Observatoire de Paris, PSL Univ., Sorbonne Univ., 77 av. Denfert-Rochereau, 75014 Paris, France

${ }^{4}$ Institute of Planetary Research, German Aerospace Center (DLR), Rutherfordstrasse 2, 12489 Berlin, Germany

5 Observatoire Astronomique de l'Université de Genève, Chemin Pegasi 51, Versoix, Switzerland

${ }^{6}$ Depto. de Astrofisica, Centro de Astrobiologia (CSIC-INTA), ESAC campus, 28692 Villanueva de la Cañada (Madrid), Spain

${ }^{7}$ Cavendish Laboratory, JJ Thomson Avenue, Cambridge CB3 0HE, UK

${ }^{8}$ Centre for Exoplanet Science, SUPA School of Physics and Astronomy, University of St Andrews, North Haugh, St Andrews KY16 9SS, UK

${ }^{9}$ Physikalisches Institut, University of Bern, Gesellsschaftstrasse 6, 3012 Bern, Switzerland

${ }^{10}$ INAF, Osservatorio Astrofisico di Catania, Via S. Sofia 78, 95123 Catania, Italy

${ }^{11}$ CFisUC, Departamento de Física, Universidade de Coimbra, 3004-516 Coimbra, Portugal

${ }^{12}$ Space Research Institute, Austrian Academy of Sciences, Schmiedlstrasse 6, 8042 Graz, Austria

${ }^{13}$ Aix Marseille Univ, CNRS, CNES, LAM, 38 rue Frédéric Joliot-Curie, 13388 Marseille, France

${ }^{14}$ Center for Space and Habitability, Gesellsschaftstrasse 6, 3012 Bern, Switzerland

15 Astrophysics Group, Keele University, Staffordshire ST5 5BG, UK

16 Department of Physics, University of Warwick, Gibbet Hill Road, Coventry CV4 7AL, UK

${ }^{17}$ Instituto de Astrofisica de Canarias, 38200 La Laguna, Tenerife, Spain

${ }^{18}$ Department of Astronomy, Stockholm University, AlbaNova University Center, 10691 Stockholm, Sweden

${ }^{19}$ Department of Space, Earth and Environment, Onsala Space Observatory, Chalmers University of Technology, 43992 Onsala, Sweden

${ }^{20}$ Departamento de Astrofisica, Universidad de La Laguna, 38206 La Laguna, Tenerife, Spain

${ }^{21}$ Institut de Ciencies de l'Espai (ICE, CSIC), Campus UAB, Can Magrans s/n, 08193 Bellaterra, Spain

${ }^{22}$ Institut d'Estudis Espacials de Catalunya (IEEC), 08034 Barcelona, Spain

23 Admatis, 5. Kandó Kálmán Street, 3534 Miskolc, Hungary

${ }^{24}$ Université Grenoble Alpes, CNRS, IPAG, 38000 Grenoble, France

${ }^{25}$ Université de Paris, Institut de physique du globe de Paris, CNRS, 75005 Paris, France

${ }^{26}$ Centre for Mathematical Sciences, Lund University, Box 118, 22100 Lund, Sweden

27 Astrobiology Research Unit, Université de Liège, Allée du 6 Août 19C, 4000 Liège, Belgium

${ }^{28}$ Space sciences, Technologies and Astrophysics Research (STAR) Institute, Université de Liège, Allée du 6 Août 19C, 4000 Liège, Belgium

${ }^{29}$ INAF, Osservatorio Astronomico di Padova, Vicolo dell'Osservatorio 5, 35122 Padova, Italy

30 Dipartimento di Fisica e Astronomia "Galileo Galilei”, Universita degli Studi di Padova, Vicolo dell'Osservatorio 3, 35122 Padova, Italy

${ }^{31}$ Leiden Observatory, University of Leiden, PO Box 9513, 2300 RA Leiden, The Netherlands

${ }^{32}$ Dipartimento di Fisica, Universita degli Studi di Torino, via Pietro Giuria 1, 10125, Torino, Italy

${ }^{33}$ University of Vienna, Department of Astrophysics, Türkenschanzstrasse 17, 1180 Vienna, Austria
} 


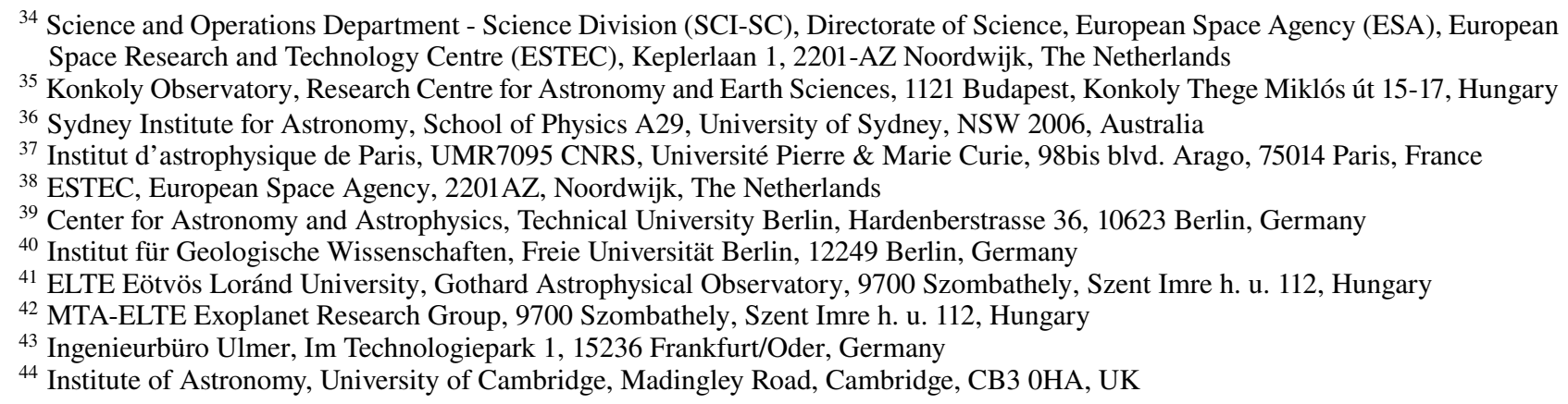

A\&A, 657, A52 (2022), https://doi.org/10.1051/0004-6361/202142196

Key words. planets and satellites: fundamental parameters - planets and satellites: composition - planets and satellites: interiors planets and satellites: individual: WASP-103b - techniques: photometric - errata, addenda

The name of co-author Sébastien Salmon (Observatoire Astronomique de l'Université de Genève, Switzerland) was missing from the author list of the original published article. It is added among the authors in this Corrigendum. 